\title{
Opinion and Special Articles: Stress when performing the first lumbar puncture may compromise patient safety
}

Mikael Johannes Vuokko Henriksen, PhD, Troels Wienecke, PhD, Jesper Kristiansen, PhD, Yoon Soo Park, PhD, Charlotte Ringsted, PhD, and Lars Konge, PhD

Neurology ${ }^{\circledR}$ 2018;90:981-987. doi:10.1212/WNL.0000000000005556

\section{Correspondence}

Dr. Henriksen

Mikael.johannes.vuokko.

henriksen@regionh.dk

\begin{abstract}
Objective

To quantify physician stress levels when performing lumbar puncture (LP) and explore operator stress effect on patient outcomes.
\end{abstract}

\section{Methods}

This was a cross-sectional, multicenter study. Novices, intermediates, and experts in performing LP were recruited from 4 departments of neurology and emergency medicine. Stress was measured before and during performance of the LP using cognitive appraisal (CA), State-Trait Anxiety Inventory-Short (STAI-S) questionnaire, and the heart rate variability measure low frequency/high frequency index (LF/HF ratio). Patient-related outcomes were pain, confidence in the operator, and postdural puncture headache (PDPH).

\section{Results}

Forty-six physicians were included in the study: 22 novices, 12 intermediates, and 12 experts. Novices had the highest stress level and experts the lowest measured by cognitive appraisal and STAI-S before and during LP performance ( $p<0.001$ for all comparisons). Novices had the highest sympathetic tonus indicated by the highest LF/HF ratio before $(p=0.004)$ and during $(p=0.056)$ LP performance. Physician stress level was not significantly related to patients' pain. However, there was a significant relationship between STAI-S during the procedure and patient confidence in the operator (regression coefficient $=-0.034, p=0.008$ ). High physician heart rate during the procedure significantly increased the odds of PDPH (odds ratio $=1.17$, $p=0.036$ ).

\section{Conclusion}

Novice stress levels were high before and during performance of LP. Stress was significantly related to patient confidence in the operator and risk of PDPH. Simulation-based training should be considered to reduce novice residents' stress levels and increase patient safety.

\footnotetext{
From the Copenhagen Academy for Medical Education and Simulation (M.J.V.H., L.K.), the Capital Region of Denmark; Faculty of Health and Medical Sciences (M.J.V.H., L.K.), University of Copenhagen; Department of Neurology (T.W.), Zealand University Hospital; The National Research Centre for the Working Environment (J.K.), Copenhagen, Denmark; Department of Medical Education (Y.S.P.), University of Illinois Chicago; and Centre for Health Science Education (C.R.), Faculty of Health, Aarhus University, Denmark.

Go to Neurology.org/N for full disclosures. Funding information and disclosures deemed relevant by the authors, if any, are provided at the end of the article.
} 


\section{Glossary}

ANOVA = analysis of variance; $\mathbf{C A}=$ cognitive appraisal; $\mathbf{C I}=$ confidence interval; $\mathrm{HR}=$ heart rate; $\mathrm{HRV}=$ heart rate variability; $\mathbf{L F} / \mathbf{H F}=$ low frequency/high frequency; LP = lumbar puncture; PDPH = postdural puncture headache; STAI-S = State-Trait Anxiety Inventory-Short.

Performing an invasive procedure on a patient can be a stressful experience for the resident, but evidence is lacking on the consequences thereof. Within neurology, a particular subject for this stress might pertain to residents' first performance of the lumbar puncture (LP).

According to the cognitive activation theory of stress, the stress sensation arises when the requirements exceed the resources for performing a given task. ${ }^{1}$ For the LP procedure, a stress sensation among novice residents might arise because the LP is a complex procedure ${ }^{2}$ with both technical and nontechnical aspects, combined with residents' uncertainties for procedural performance ${ }^{3}$ and fear of doing harm. ${ }^{4}$

The consequences of being in a state of acute mental stress are reduced working memory, decreased psychomotor performance, ${ }^{5}$ and impaired performance. ${ }^{6}$ These negative effects of acute stress might therefore compromise patient safety. ${ }^{7}$ However, for nonsurgical invasive procedures such as the LP, there is a lack of evidence regarding measurements of acute mental stress across operators' experience levels and how stress might influence the performance and patient-related outcomes. Residents' stress levels and the effect on procedural performance and patient safety should be explored deeper as novices are more prone to the negative effects of stress.

Hence, the aims of this study were to explore acute mental stress levels across operator LP experience levels and the relation between operator stress and patient outcomes.

\section{Methods}

This was a cross-sectional, multicenter study and reported according to the STROBE (Strengthening the Reporting of Observational Studies in Epidemiology) guidelines. ${ }^{8}$

Based on expert agreement, we defined 3 groups of experience levels in LP performance: (1) novices, (2) intermediates, and (3) experts. Inclusion criteria for each of the groups were as follows: (1) medical doctor or medical student; no previous LP experience. (2) Medical doctor or medical student; performing LP regularly; having an LP experience of 10 to 80 procedures. (3) Consultant, performing LP regularly; supervising junior LP operators; having 100 or more LP experiences. Physicians with experience in the gaps between these groups were not included. We recruited participants from 3 departments of neurology and one emergency department in Denmark. The recruitment period was December 2016 to June 2017.
We followed the recommendations to combine stress-level measurements exploring both the subjective (cognitive and emotional) stress sensation and the physiologic response. ${ }^{7}$

Cognitive appraisal (CA) is a predictor of stress response under high acuity conditions with significant correlation to cortisol responses. ${ }^{9}$ According to CA theory, an individual facing a threatening situation first assesses the demands for handling the situation (primary appraisal). The primary appraisal will be influenced by related experience and personal expectations to the outcome of the situation. ${ }^{1}$ Subsequently, the individual determines the resources available for the situation (secondary appraisal). ${ }^{9}$ Primary appraisal was examined by asking the participants: "How demanding do you feel the upcoming LP to be?" Secondary appraisal was examined by asking: "How capable are you to handle this LP?" For both questions, the participants rated their answers on an anchored 6-point Likert scale. A CA was calculated as a ratio of primary vs secondary appraisal. If the perceived resources exceeded the demands, the CA ratio would be $<1$ and classified as a challenge. If the demands equaled or outweighed the resources, the CA ratio would be $\geq 1$ and classified as a threat. ${ }^{10}$

The instrument State-Trait Anxiety Intervention-Short (STAI-S) was selected as another indicator of subjective stress. The STAI-S has been used and recommended for assessing emotional response during performance of a medical procedure. ${ }^{7,11,12}$

STAI-S includes 6 items (e.g., "I am tense"), each rated on a 4-point anchored Likert scale. ${ }^{13}$ Minimum score was 6 and maximum score was 24 , indicating great anxiety.

Heart rate variability (HRV) is a recording of the participant's $\mathrm{R}-\mathrm{R}$ interval of an ECG. This interval is highly correlated to the nervous system and reflects sympathetic or parasympathetic contributions to cardiac rhythm modulation. ${ }^{14,15}$

For the HRV recordings, we used a Faros $180^{\circ}$ sensor (Mega Electronics Ltd., Kuopio, Finland). The sensor was set to 500$\mathrm{Hz}$ continuous recordings. We used the software Kubios HRV version $2.2^{16}$ (Biosignal Analysis and Medical Group, Kuopio, Finland) for the HRV analysis. We extracted the HRV timedomain parameter of heart rate (HR) and, for frequency analysis, the low frequency/high frequency (LF/HF) ratio reflecting the global sympathico-vagal balance. ${ }^{17}$ In accordance with contemporary recommendations, we chose shortterm recordings of 5 minutes. ${ }^{17}$ 
Stress was measured at 2 predefined time points: (1) preperformance, 5 minutes before performance (CA, STAI-S, and HRV); and (2) during procedure; STAI-S was obtained when the participant asked for the needle. HRV was registered continuously, and the value 5 minutes before obtaining CSF or abandoning the procedure was used as the "during procedure" measurement. The following patient outcomes were recorded during the procedure: number of needle insertions, CSF successfully obtained, and needle insertion time. After the procedure, the patients rated their procedure-related pain and their confidence in the operator on anchored 10-point Likert scales.

Postdural puncture headache $(\mathrm{PDPH})$ was registered by telephone interview by M.H. 7 days after the procedure. The assessment of PDPH followed Lybecker criteria: (1) history of LP, (2) headache severity compared to previous headaches, and (3) postural component. ${ }^{18}$ To avoid bias associated with knowledge of experience level, all patients with any headache were discussed with T.W. (expert neurologist), who was blinded to operator experience level. T.W. had the final decision of PDPH classification.

\section{Study setting}

The LP procedures were performed at the outpatient clinic at all 3 departments of neurology. The participants performed the procedure as part of ordinary clinical practice using local equipment. To avoid bias, M.H. was the assistant for all operators during all procedures in this study. When the LP was performed by a novice, a supervisor was on-site. Patient inclusion criteria were as follows: referred for an LP; Glasgow Coma Scale score of 15; proficiency in the Danish language; age between 18 and 80 years; understanding of study implications and cooperation without a need for personal assistance; and written and orally informed consent provided. Exclusion criteria included LP on suspicion of dementia diagnosis, cognitive impairment assessed by the study investigator or local doctor, and physical disability requiring personal assistance.

Physicians completed the pre-procedure stress measures without knowledge of the patient's body habitus, sex, or underlying medical history. To avoid bias of the HRV measurements caused by differences in movement between the pre-performance and during procedure HRV recording, the operators performed minor physical tasks, such as operating their computer in a sitting position, to ensure movements at a level equal to the needle insertion. During the performance, physicians were shown the STAI-S questions on a sheet of paper, answered the study investigator using only numbers, and were blinded to patient identity.

\section{Standard protocol approvals, registrations, and patient consents}

The local ethics committee of Capital Region Denmark ruled that approval was not required for this study (protocol 16040848). The study was reported on clinicaltrials.gov (identifier: NCT03192423). All participants_both physicians and patients - were informed verbally and in writing of the study's purpose and that it was voluntary and entirely anonymous. All participants completed written informed consent.

\section{Statistics}

Previous similar studies using stress measures identified meaningful differences with sample sizes of 10 to 18 participants, ${ }^{11,19,20}$ and hence, we aimed to include more than 10 in the expert and intermediate groups and more than 20 in the novice group.

We determined possible differences in patient characteristics by groups using analysis of variance (ANOVA) for age and $\chi^{2}$ test for sex and premedication. For analysis of the stress measurements, we log-transformed the LF/HF ratio data as these were nonnormally distributed ( $\mathrm{LnLF} / \mathrm{HF}$ ratio). We compared data across all 3 groups using ANOVA. Post hoc tests using Bonferroni adjustments explored differences between the individual groups. Because age was a confounder for the HRV analysis, we performed a post hoc independent $t$ test to examine differences in HRV data between the intermediate and novice groups. For analysis of changes in HR between pre-performance and during performance, we used paired $t$ test individually for all 3 groups.

Bias analyses were conducted using ANOVA to examine possible differences in distribution between groups for patient-related factors, ${ }^{21-23}$ including needle size, number of needle insertions, patient body mass index, volume of CSF, and positioning. Kruskal-Wallis test was used for exploring needle insertion time across all groups as this variable was nonnormally distributed.

We conducted multiple regression analyses of physician stress variables predicting patient confidence in the operator as a dependent variable. We performed a $3 \times 2$ contingency table of the 3 experience levels, whether the procedure had failed (yes or no), and used the Spearman correlation test to explore whether the distribution was significantly different from chance.

We used multiple logistic regression to examine the effect of physician stress on the binary outcome of patients' risk of developing PDPH. To facilitate interpretation, odds ratios were used to report the regression results by exponentiating the coefficient from the logistic regression. This analysis was done across all 3 groups and within the novice group as PDPH only appeared among patients in this group. For all regression analyses, we checked for possible multicollinearity among the independent variables.

\section{Results}

Forty-six physicians were included, representing 22 in the novice group, 12 in the intermediate group, and 12 in the expert group, performing one procedure each, resulting in 46 
procedures for analysis. Baseline characteristics and missing variables of the participating physicians and patients are presented in table 1. Participants in the intermediate and expert groups reported performing 10 to 20 LPs annually.

A limited number of patients were excluded or refused participation, primarily because of suspicion of Alzheimer disease; these patients had their LP performed by an operator outside this study.

\section{Stress measurements}

The CA scores were 1.27 (SD 0.45), 0.68 (SD 0.33), and 0.39 (SD 0.22) for novices, intermediates, and experts, respectively $(p<0.001)$. Post hoc analysis showed significant differences between novices and intermediates $(p<0.001)$ and novices and experts $(p<0.001)$ but not between intermediates and experts $(p=0.210)$.

The pre-performance STAI-S scores were 12.6 points (SD 2.24), 10.5 points (SD 2.7), and 7.6 points (SD 2.1) for novices, intermediates, and experts, respectively $(p<0.001)$. The post hoc analysis showed significant differences between novices and experts $(p<0.001)$ and intermediates and experts $(p=$ $0.022)$ but not between novices and intermediates $(p=0.052)$.

The STAI-S scores during performance were 13.2 (SD 2.75), 10.9 (SD 2.5), and 7.58 (SD 2.1) for novices, intermediates, and experts, respectively $(p<0.001)$. The post hoc analysis showed significant differences between novices and intermediates, novices and experts, and intermediates and experts $(p=0.047$, $p<0.001$, and $p=0.012$, respectively).

For HR, we found no differences between the 3 groups preperformance $(p=0.440)$, but there was a significant difference during the procedure $(p=0.034)$. Post hoc tests demonstrated a significant difference between experts and intermediates $(p=$ $0.029)$ but not between experts and novices $(p=0.24)$ or between novices and intermediates $(p=0.48)$. The HR increased significantly from pre-performance to during performance for both the intermediate and novice groups $(p<0.001$ for both groups) but not the expert group $(p=0.08)$.

For pre-performance LnHF/LF ratio, we found a significant difference between the 3 groups $(p=0.004)$. The intermediate group had the lowest sympathetic level, and post hoc tests demonstrated a significant difference compared to the novices $(p=0.014)$ and experts $(p=0.012)$. There was no significant difference between the groups in $\mathrm{LnHF} / \mathrm{LF}$ ratio during the procedure $(p=0.058)$. Table 2 presents details of the physician stress measurements.

\section{Patient-related outcomes}

No patients received additional LPs in the time from study participation to the telephone interview. There was no difference between the 3 groups regarding the volumes of tapped CSF $(p=0.486)$ or number of needle insertions $(p=0.609)$. Novices used significantly more time than intermediate and expert operators $(p=0.028)$.

Table 1 Baseline characteristics of physicians and patients

\begin{tabular}{|c|c|c|c|c|}
\hline & Expert group & Intermediate group & Novice group & $p$ Value \\
\hline \multicolumn{5}{|l|}{ Physician characteristics } \\
\hline No. & 12 & 12 & 22 & \\
\hline Age, y & $48.3(7.5)^{a}$ & $29.4(3.7)$ & $28.3(4.3)^{\mathrm{b}}$ & \\
\hline Range & $37-65$ & $26-39$ & $25-40$ & \\
\hline Sex, M/F & $11 / 1$ & $2 / 10$ & $8 / 14$ & \\
\hline No. of LPs performed & $383(376)$ & $41.4(26.2)$ & & \\
\hline \multicolumn{5}{|l|}{ Patient characteristics } \\
\hline Missing data within groups & $5^{c}$ & 0 & $1^{d}$ & \\
\hline Age, y & $56.8(16.1)$ & $50.5(12.9)$ & $46.4(12.4)$ & 0.12 \\
\hline Sex, M/F & $4 / 8$ & $7 / 5$ & $13 / 8$ & 0.91 \\
\hline BMI & $24.4(3)$ & $26.4(3.2)$ & $26.6(6.0)$ & 0.53 \\
\hline Premedication with analgesia, yes/no & $4 / 8$ & $1 / 11$ & $7 / 14$ & 0.25 \\
\hline
\end{tabular}

Abbreviations: $\mathrm{BMI}=$ body mass index; LnLF/HF = natural logarithm low frequency/high frequency; LP = lumbar puncture.

Data are count or mean (SD). Patient characteristics: one-way analysis of variance was used to compare for differences between the groups. Pearson $\chi^{2}$ test was used for distribution of sex and premedication.

a Analysis of variance test was used to compare differences in age between all 3 groups $(p<0.001)$.

${ }^{\mathrm{b}}$ Post hoc test of difference in age between intermediate and novice groups by independent sample $t$ test ( $\left.p=0.48\right)$.

c Data on heart rate and LnLF/HF ratio are missing for 5 expert physicians.

${ }^{\mathrm{d}}$ Data on premedication missing for one patient. 
Table 2 Stress measurements in physicians

\begin{tabular}{|c|c|c|c|c|}
\hline Stress measurements & Expert group & Intermediate group & Novice group & $p$ Value \\
\hline \multicolumn{5}{|l|}{ Pre-performance } \\
\hline Cognitive appraisal & $0.39(0.22)^{a}$ & $0.68(0.33)$ & $1.27(0.45)^{c}$ & $<0.001$ \\
\hline STAI-S & $7.58(2.1)^{a}$ & $10.5(2.71)^{\mathrm{b}}$ & $12.59(2.24)$ & $<0.001$ \\
\hline Heart rate & $76.9(14.4)$ & $85.9(14.3)$ & $83.5(14.9)^{c}$ & 0.440 \\
\hline LnLF/HF ratio & $1.69(0.89)$ & $0.54(0.59)^{b}$ & $1.16(0.7)^{c}$ & 0.004 \\
\hline \multicolumn{5}{|l|}{ During performance } \\
\hline STAI-S & $7.58(1.51)^{\mathrm{a}}$ & $10.91(2.51)^{\mathrm{b}}$ & $13.18(2.75)^{\mathrm{c}}$ & $<0.001$ \\
\hline Heart rate & $80.2(13.8)$ & $99.0(15.4)^{\mathrm{b}}$ & $91.5(14.2)$ & 0.034 \\
\hline LnLF/HF ratio & $1.70(0.74)$ & $1.08(0.55)$ & $1.45(0.51)$ & 0.058 \\
\hline
\end{tabular}

Abbreviations: $\mathrm{LnLF} / \mathrm{HF}=$ natural logarithm low frequency/high frequency; STAI-S = State-Trait Anxiety Inventory-Short.

Data are mean (SD). Comparison of stress measures in physicians across the 3 groups.

a Significant difference between expert group and novice group.

${ }^{\mathrm{b}}$ Significant difference between expert group and intermediate group.

c Significant difference between intermediate group and novice group.

Failure to obtain CSF was experienced by 9 operators in the novice group, 2 in the intermediate group, and 1 in the expert group $(p=0.027)$. There were no differences in patient pain scores between the 3 groups $(p=0.70)$. Local anesthesia was used by $77 \%$ in the novice group, $66 \%$ in the intermediate group, and $33 \%$ in the expert group, which was a significant difference $(p=0.038)$.

The scores for patient confidence in the operator were 7.24 (SD 2.7), 8.75 (SD 2.4), and 9.25 (SD 1.1) for novices, intermediates, and experts, respectively $(p=0.043)$. The post hoc analysis did not identify significant differences between 2 individual groups. Table 3 presents a description of the patient outcomes.
There was a significant relationship between physician stress and patient confidence. For every unit increase in STAI-S during-procedure score, patient confidence decreased by 0.34 units $(p=0.008)$. Operator HR and LnLF/HF ratio did not have a significant relationship with patient confidence using the same multiple regression model $(p=0.889$ and $p=0.839$, respectively).

Eight patients developed $\mathrm{PDPH}$; all procedures were performed by an operator from the novice group $(p=0.004)$. The procedure was completed by the novice operator in 4 patients, and a supervisor performed additional needle insertions to obtain CSF in the remaining patients. Using

Table 3 Patient-related lumbar puncture outcomes across operator experience levels

\begin{tabular}{|c|c|c|c|c|}
\hline & Expert group $(n=12)$ & Intermediate group $(n=12)$ & Novice group $(n=22)$ & $p$ Value \\
\hline Tapped volume of CSF, mL & $4.9(2.0)$ & $6.2(3.7)$ & $5.8(1.8)$ & 0.49 \\
\hline No. of needle insertions by operator & $1.42(1.0)$ & $1.25(0.45)$ & $1.18(0.50)$ & 0.61 \\
\hline Range & $1-4$ & $1-2$ & $0-2$ & \\
\hline Total no. of needle insertions & $1.42(1.0)$ & $1.42(0.7)$ & $1.68(0.95)$ & 0.61 \\
\hline Range & $1-4$ & $1-3$ & $1-4$ & \\
\hline Needle insertion time, min & $02: 29(03: 40)$ & $02: 23(02: 17)$ & 03:55 (02:33) & 0.028 \\
\hline Range & $00: 17-13: 00$ & $00: 21-06: 56$ & 00:59-09:39 & \\
\hline No. of failed procedures & 1 & 2 & 9 & 0.027 \\
\hline No. of operators applying local anesthetic & 4 & 8 & 17 & 0.038 \\
\hline Patient-experienced pain intensity & $3.58(2.39)$ & $4.50(2.61)$ & $3.95(2.89)$ & 0.704 \\
\hline Patient confidence in operator & 9.25 (1.14) & $8.75(2.4)$ & $7.24(2.7)$ & 0.043 \\
\hline
\end{tabular}

Data are count or mean (SD). Total number of needle insertions includes both initial operator and called-in supervisor. Patient-experienced pain and patient confidence were rated on 10-point Likert scales. 
logistic regression across all groups, we found that for each unit increase in CA, the odds of developing $\mathrm{PDPH}$ was 5.06 greater (95\% confidence interval [CI] 1.06-24.19, $p=0.042$ ). For each unit increase in pre-performance STAI-S, the odds of developing PDPH was 1.56 (95\% CI 1.08-2.25) times greater $(p=0.017)$. Analysis within the novice group demonstrated that increased operator HR during performance significantly increased the odds of patients developing PDPH $(p=0.036)$ (odds ratio $=1.17,95 \%$ CI 1.01-1.36).

\section{Discussion}

This study contributes to the literature by exploring the significance of stress to clinical outcomes and not only simulated performance outcomes. ${ }^{19}$ We found that novice operators had a significantly higher subjective and physiologic stress level, in particular before performance, compared to intermediate and expert operators. Novices' HR during the procedure was an independent risk factor for their patients developing PDPH.

CA ratio in novices exceeded 1 , indicating that they appraised their first LP performance as threat level. ${ }^{10}$ This finding aligns with the significantly higher STAI-S score both preperformance and during performance, reflecting that novices hold an affective response to performing LP. We further identified that novices were dominated by higher sympathetic tonus pre-performance indicated by the significantly higher $\mathrm{LnLF} / \mathrm{HF}$ ratio compared to the intermediates. Of note, the post hoc analysis additionally identified that intermediates had a lower LnLF/HF ratio compared to experts. This might be explained by the differences between the expert group and the other groups in age and ratio of male/female participants. ${ }^{24}$

Stress measurements in expert operators were stable from pre-performance to during performance. By contrast, novice and intermediate operators experienced increased stress levels during performance. In essence, the results indicate that novice LP operators are highly susceptible to subjective and physiologic stress responses. Pre-performance stress in novices may be attributable to uncertainty about the procedure and a lack of competence, $3,25,26$ combined with a fear of causing patient harm. ${ }^{4}$ According to the cognitive activation theory of stress, such situations could easily give rise to a stress response $^{1}$ that could explain residents' tendency to avoid performing the procedure. ${ }^{27}$ Furthermore, the results indicate that intermediate performers also experience a considerable increase in stress during the procedure, which could be attributed to the absence of a supervisor.

The clinical consequences of physicians experiencing a stress state are not well explored. Nonetheless, a stress state impairs psychomotor performance, ${ }^{5,11}$ decision-making, ${ }^{28,29}$ communication, ${ }^{29}$ and retrieval of declarative memory. ${ }^{30}$ Furthermore, physicians with a high CA for complex situations will perform with significantly impaired nontechnical performance. ${ }^{19}$ This is undesirable for the LP procedure because it requires the operator to integrate nontechnical aspects related to patient communication. ${ }^{31}$ This might explain our finding of reduced confidence in the operator across all experience levels correlated to the operator's STAI-S score during the procedure. This relation was unaffected by operator age.

There was a significant increased risk for the patient to develop $\mathrm{PDPH}$ if the operator had an increased preperformance CA or STAI-S. However, in our study, only patients whose procedure was performed by a novice operator developed PDPH, which limits the ability to conduct across experience level statistical analyses of correlations between stress and PDPH. Nonetheless, when adjusting for experience level, novice operators with increased HR during the performance significantly increased patient risk of PDPH. Because we adjusted for patient characteristics and other previously identified risk factors for PDPH, we believe that this finding reflects another potential risk factor for the riddle of PDPH. The results of this study demonstrating that physician stress reduces patient safety and confidence warrant more awareness of the mental stress of residents performing invasive procedures. In particular, further research on strategies to reduce physician stress level should be considered in an effort to optimize patient confidence and safety. Studies have not indicated any potential beneficial effect of experiencing a stress state before performing a technical procedure. In contrast, evidence suggests that negative emotions, such as stress and anxiety, impair processing of information in a way that would hinder optimal performance. ${ }^{32}$

Previous studies have identified that novices and even more experienced residents improve their performance and decrease their anxiety by simulation-based training. ${ }^{25,28}$ However, the effects of simulation-based training on stress and performance in the clinical setting remain to be demonstrated for the LP procedure.

There are some limitations to this study. We were unable to obtain data on patient indications for LP and preceding use of anxiolytics. Nonetheless, the inclusion criteria ensured that patients were homogeneous in clinical condition and appearance. Our criteria for the groups were based on expert consensus. Participants in the intermediate group were independent operators, reflecting a level of competence but they were not expert operators. Therefore, we believe the criterion reflects the intention of our aim, but this is a subject for future studies.

We did not use an objective assessment of the LP performance. Future studies should investigate correlation of stress levels to objective performance scores, including patient outcomes. ${ }^{7}$ Also, the expert group differed from the other groups in age and male/female ratio. This limits the possibility of drawing conclusions regarding the LnLF/HF differences across experience levels. Unfortunately, for practical reasons, it was not possible to obtain a resting measurement of HRV, which may have contributed to elucidation of possible 
differences between the groups in an unstressed state. Another limitation is the omission of cortisol level measurement to explore the hypothalamic-pituitary-adrenal axis. Salivary samples were not feasible in our setup.

This study identified that novice LP operators hold a high stress level, in particular before their performance on their first patient. Novices have a higher rate of failing the procedure, and patients of notably stressed novices have a higher incidence of experiencing PDPH.

To optimize patient safety, these findings warrant more research in effective training strategies to increase operator competence before performing LP procedures on patients.

\section{Author contributions}

Mikael Johannes Vuokko Henriksen: study concept and design, acquisition of data, analysis and interpretation of data, critical revision of manuscript for intellectual content. Troels Wienecke: study concept and design, acquisition of data, critical revision of manuscript for intellectual content. Jesper Kristiansen: study concept and design, analysis and interpretation of data, critical revision of manuscript for intellectual content. Yoon Soo Park: analysis and interpretation of data, critical revision of manuscript for intellectual content. Charlotte Ringsted: study concept and design, analysis and interpretation of data, critical revision of manuscript for intellectual content, study supervision. Lars Konge: study concept and design, analysis and interpretation of data, critical revision of manuscript for intellectual content, study supervision.

\section{Study funding}

Partly supported by the Danish NGO TrygFonden (ID 105112).

\section{Disclosure}

The authors report no disclosures relevant to the manuscript. Go to Neurology.org/N for full disclosures.

\section{References}

1. Ursin H, Eriksen HR. The cognitive activation theory of stress. Psychoneuroendocrinology 2004;29:567-592.

2. Roos KL. Lumbar puncture. Semin Neurol 2003;23:105-114.

3. Dehmer JJ, Amos KD, Farrell TM, Meyer AA, Newton WP, Meyers MO. Competence and confidence with basic procedural skills: the experience and opinions of fourth-year medical students at a single institution. Acad Med 2013;88:682-687.

4. Henriksen MJV, Wienecke T, Thagesen $\mathrm{H}$, et al. Assessment of residents readiness to perform lumbar puncture: a validation study. J Gen Intern Med 2017;32:610-618.

5. Bajunaid K, Mullah MAS, Winkler-Schwartz A, et al. Impact of acute stress on psychomotor bimanual performance during a simulated tumor resection task. J Neurosurg 2017;126:71-80
6. LeBlanc VR. The effects of acute stress on performance: implications for health professions education. Acad Med 2009;84:S25-S33.

7. Arora S, Sevdalis N, Nestel D, Woloshynowych M, Darzi A, Kneebone R. The impact of stress on surgical performance: a systematic review of the literature. Surgery 2010; 147:318-330.e6.

8. von Elm E, Altman DG, Egger M, et al. The Strengthening the Reporting of Observational Studies in Epidemiology (STROBE) statement: guidelines for reporting observational studies. Lancet 2007;370:1453-1457.

9. Harvey A, Nathens AB, Bandiera G, LeBlanc VR. Threat and challenge: cognitive appraisal and stress responses in simulated trauma resuscitations. Med Educ 2010;44: 587-594.

10. Tomaka J, Blascovich J, Kibler J, Ernst JM. Cognitive and physiological antecedents of threat and challenge appraisal. J Pers Soc Psychol 1997;73:63-72.

11. Arora S, Sevdalis N, Aggarwal R, Sirimanna P, Darzi A, Kneebone R. Stress impairs psychomotor performance in novice laparoscopic surgeons. Surg Endosc 2010;24: 2588-2593.

12. Jones KI, Amawi F, Bhalla A, Peacock O, Williams JP, Lund JN. Assessing surgeon stress when operating using heart rate variability and the State Trait Anxiety Inventory: will surgery be the death of us? Colorectal Dis 2015;17:335-341.

13. Marteau TM, Bekker H. The development of a six-item short-form of the state scale of the Spielberger State-Trait Anxiety Inventory (STAI). Br J Clin Psychol 1992;31: 301-306.

14. Cygankiewicz I, Zareba W. Heart rate variability. In: Bujis RM, Swaab DF, editors. Handbook of Clinical Neurology, Vol 11 (3rd series). Amsterdam: Elsevier B.V.; 2013:379-394.

15. Castaldo R, Melillo P, Bracale U, Caserta M, Triassi M, Pecchia L. Acute mental stress assessment via short term HRV analysis in healthy adults: a systematic review with meta-analysis. Biomed Signal Process Control 2015;18:370-377.

16. Tarvainen MP, Niskanen JP, Lipponen JA, Ranta-Aho PO, Karjalainen PA. Kubios HRV: heart rate variability analysis software. Comput Methods Programs Biomed 2014;113:210-220.

17. Ernst G. Heart Rate Variability. London: Springer; 2014.

18. Lybecker H, Djernes M, Schmidt JF. Postdural puncture headache (PDPH): onset, duration, severity, and associated symptoms. An analysis of 75 consecutive patients with PDPH. Acta Anaesthesiol Scand 1995;39:605-612.

19. Harvey A, Bandiera G, Nathens AB, LeBlanc VR. Impact of stress on resident performance in simulated trauma scenarios. J Trauma Acute Care Surg 2012;72:497-503.

20. Böhm B, Rötting N, Schwenk W, Grebe S, Mansmann U. A prospective randomized trial on heart rate variability of the surgical team during laparoscopic and conventional sigmoid resection. Arch Surg 2001;136:305-310.

21. Evans RW, Armon C, Frohman EM, Goodin DS. Assessment: prevention of postlumbar puncture headaches: report of the Therapeutics and Technology Assessment Subcommittee of the American Academy of Neurology. Neurology 2000;55:909-914.

22. Monserrate AE, Ryman DC, Ma S, et al. Factors associated with the onset and persistence of post-lumbar puncture headache. JAMA Neurol 2015;72:325-332.

23. van Oosterhout WPJ, van der Plas AA, van Zwet EW, Zielman R, Ferrari MD, Terwindt GM. Postdural puncture headache in migraineurs and nonheadache subjects: a prospective study. Neurology 2013;80:941-948

24. Antelmi I, de Paula RS, Shinzato AR, Peres CA, Mansur AJ, Grupi CJ. Influence of age, gender, body mass index, and functional capacity on heart rate variability in a cohort of subjects without heart disease. Am J Cardiol 2004;93:381-385.

25. Barsuk JH, Cohen ER, Caprio T, McGaghie WC, Simuni T, Wayne DB. Simulationbased education with mastery learning improves residents' lumbar puncture skills. Neurology 2012;79:132-137.

26. Lammers RL, Temple KJ, Wagner MJ, Ray D. Competence of new emergency medicine residents in the performance of lumbar punctures. Acad Emerg Med 2005; 12:622-628.

27. Kneen R. The role of lumbar puncture in suspected CNS infection: a disappearing skill? Arch Dis Child 2002;87:181-183.

28. Arora S, Aggarwal R, Moran A, et al. Mental practice: effective stress management training for novice surgeons. J Am Coll Surg 2011;212:225-233.

29. Wetzel CM, Kneebone RL, Woloshynowych M, et al. The effects of stress on surgical performance. Am J Surg 2006;191:5-10.

30. Kirschbaum C, Wolf OT, May M, Wippich W, Hellhammer DH. Stress- and treatment-induced elevations of cortisol levels associated with impaired declarative memory in healthy adults. Life Sci 1996;58:1475-1483.

31. Engelborghs S, Niemantsverdriet E, Struyfs $\mathrm{H}$, et al. Consensus guidelines for lumbar puncture in patients with neurological diseases. Alzheimers Dement 2017;8:111-126.

32. McMillan $\mathrm{HJ}$, Writer $\mathrm{H}$, Moreau KA, et al. Lumbar puncture simulation in pediatric residency training: improving procedural competence and decreasing anxiety. BMC Med Educ 2016;16:198. 


\section{Neurology}

\section{Opinion and Special Articles: Stress when performing the first lumbar puncture may compromise patient safety}

Mikael Johannes Vuokko Henriksen, Troels Wienecke, Jesper Kristiansen, et al. Neurology 2018;90;981-987

DOI 10.1212/WNL.0000000000005556

This information is current as of May 21, 2018

\section{Updated Information \& Services}

References

Citations

Subspecialty Collections

Permissions \& Licensing

Reprints including high resolution figures, can be found at: http://n.neurology.org/content/90/21/981.full

This article cites 30 articles, 4 of which you can access for free at: http://n.neurology.org/content/90/21/981.full\#ref-list-1

This article has been cited by 2 HighWire-hosted articles: http://n.neurology.org/content/90/21/981.full\#\#otherarticles

This article, along with others on similar topics, appears in the following collection(s):

Clinical trials Observational study (Cohort, Case control)

http://n.neurology.org/cgi/collection/clinical_trials_observational_study cohort_case_control

Error in medicine

http://n.neurology.org/cgi/collection/error_in_medicine

Other Education

http://n.neurology.org/cgi/collection/other_education

Patient safety

http://n.neurology.org/cgi/collection/patient_safety

Information about reproducing this article in parts (figures,tables) or in its entirety can be found online at:

http://www.neurology.org/about/about_the_journal\#permissions

Information about ordering reprints can be found online:

http://n.neurology.org/subscribers/advertise

Neurology ${ }^{\circledR}$ is the official journal of the American Academy of Neurology. Published continuously since 1951, it is now a weekly with 48 issues per year. Copyright (O) 2018 American Academy of Neurology. All rights reserved. Print ISSN: 0028-3878. Online ISSN: 1526-632X.

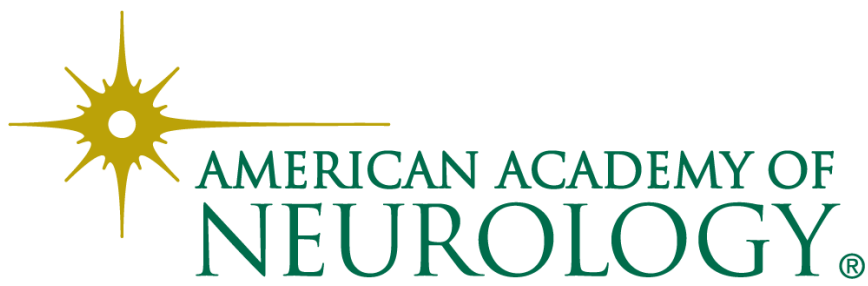

